\title{
ON ENTIRE FUNCTIONS WITH GAP POWER SERIES
}

\author{
by J. M. ANDERSON and K. G. BINMORE
}

(Received 23 June, 1969; revised 15 June, 1970)

1. Introduction. In this note we consider transcendental entire functions

$$
f(z)=\sum_{n=0}^{\infty} a_{n} z^{n}
$$

whose power series contain gaps, i.e.

$$
a_{n}=0 \quad(n \notin \Lambda),
$$

where $\Lambda=\left\{\lambda_{k}\right\}$ is a suitable set of positive integers. We denote the set of all such functions $f(z)$ by $E(\Lambda)$. As usual $M(r)=M(r, f)$ denotes the maximum modulus of $f(z)$ on the circle $|z|=r$. The order $\rho$ and the lower order $\lambda$ of $f(z)$ are defined by

$$
\begin{aligned}
& \rho=\underset{r \rightarrow \infty}{\limsup } \frac{\log \log M(r)}{\log r}, \\
& \lambda=\liminf _{r \rightarrow \infty} \frac{\log \log M(r)}{\log r},
\end{aligned}
$$

respectively.

The following theorem is due to Macintyre [4].

THEOREM A. Suppose that $f(z) \in E(\Lambda)$, where

$$
\sum_{k=1}^{\infty} \lambda_{k}^{-1}<\infty
$$

Then $f(z)$ is unbounded on $z>0$.

Edrei [2] has shown that, if the order of $f(z)$ is taken into account, then the gap condition (3) may be relaxed. He proves

THEOREM B. Suppose that $f(z) \in E(\Lambda)$ and is of finite order $\rho$, and that

$$
\liminf _{s \rightarrow \infty} \frac{1}{\log s} \sum_{\lambda_{n} \leqq s} \lambda_{n}^{-1}<\frac{1}{2 \rho} .
$$

Then $f(z)$ is unbounded on $z>0$.

From both theorems we may draw the further conclusion that $f(z)$ has no finite radial asymptotic values. Both Macintyre and Edrei use an idea of Pólya [6] to show that, even for this weaker conclusion, their gap conditions are best possible. More precisely, Macintyre shows that, if $\Lambda$ is any set of positive integers for which (3) does not hold, then there exists an 
$f(z) \in E(\Lambda)$ such that $f(x) \rightarrow 0$ as $x \rightarrow+\infty$. Similarly, Edrei shows that, if $\Lambda$ is any set of positive integers for which (4) does not hold, then there exists an $f(z) \in E(\Lambda)$ of finite order $\rho$ such that $f(x) \rightarrow 0$ as $x \rightarrow+\infty$.

In this note we prove a result which contains those of Macintyre and Edrei quoted above. We define

$$
\phi(s)=\phi(s, f)=\log M\left(e^{s}\right) .
$$

It is well-known that $\phi(s)$ is a convex function of $s$. We shall suppose that $\psi(s)$ is also a convex function of $s$ which satisfies

$$
\frac{\psi(s)}{s} \rightarrow+\infty \quad(s \rightarrow+\infty)
$$

and define

$$
\tau_{\psi}(s)=\max _{t}\{s t-\psi(t)\}
$$

The functions $\psi(s)$ and $\tau_{\psi}(s)$ display a certain duality in so far as $\tau_{\psi}(s)$ is a convex function of $s$ which satisfies a relation corresponding to (6) and, moreover,

$$
\psi(s)=\max _{t}\left\{s t-\tau_{\psi}(t)\right\}
$$

see $[5$, p. 7]. We then have

THEOREM 1. Let $\Lambda$ be a set of positive integers. Then a necessary and sufficient condition that every $f(z) \in E(\Lambda)$ which satisfies

$$
\phi(s, f)=O(\psi(s)) \quad(s \rightarrow+\infty)
$$

be unbounded on $z>0$ is that

$$
\liminf _{s \rightarrow \infty}\left\{2 \sum_{\lambda_{n} \leqq s} \lambda_{n}^{-1}-\frac{\tau_{\psi}(s)}{s}\right\}=-\infty .
$$

We define the $\psi$-order $\rho(0 \leqq \rho \leqq \infty)$ of an entire function $f(z)$ by

$$
\rho=\limsup _{s \rightarrow \infty} \frac{\psi^{-1}(\phi(s))}{s} .
$$

The $\psi$-order reduces to the usual notion of order in the case when $\psi$ is the exponential function. We can also define the lower $\psi$-order of $f(z)$ in a similar way. We then have

COROLlaRY 1. Let $\Lambda$ be a set of positive integers. Then a sufficient condition that every $f(z) \in E(\Lambda)$ which is of finite $\psi$-order at most $\rho$ be unbounded on $z>0$ is that

$$
\liminf _{s \rightarrow \infty} \frac{1}{s_{\lambda_{n} \leqq \psi(s)}} \lambda_{n}^{-1}<\frac{1}{2 \rho} .
$$


If $\log \psi(s)$ is a convex function of $s$, the gap condition is also necessary.

COROLlaRY 2. Let $\Lambda$ be a set of positive integers. A sufficient condition that every $f(z) \in E(\Lambda)$ which is of finite lower $\psi$-order at most $\lambda$ be unbounded on $z>0$ is that

$$
\limsup _{s \rightarrow \infty} \frac{1}{s_{\lambda_{n}} \leqq \psi(s)} \lambda_{n}^{-1}<\frac{1}{2 \lambda} .
$$

If $\log \psi(s)$ is a convex function of $s$, the gap condition is also necessary.

In the case when $\psi(s)=\exp s$, Corollary 1 reduces to Edrei's Theorem B and Corollary 2 to a companion theorem. In [2], Edrei noted that his results, although best possible, contain an imprecision with regard to the type of entire function considered. Such an imprecision is inevitable in the case of Theorem $\mathrm{B}$, since $f(z)$ is bounded on $z>0$ if and only if $f(R z)(R>0)$ is bounded on $z>0$. However, Edrei's result can still be sharpened somewhat by taking into account the type of $f(z)$. As usual, if $f(z)$ is of finite order $\rho$, its type $\tau$ is defined by

$$
\tau=\limsup _{r \rightarrow \infty} \frac{\log M(r)}{r^{\rho}} .
$$

COROLlary 3. Let $\Lambda$ be a set of positive integers. A necessary and sufficient condition that every $f(z) \in E(\Lambda)$ which is of finite order at most $\rho$ and finite type be unbounded on $z>0$ is that

$$
\liminf _{s \rightarrow \infty}\left\{\sum_{\lambda_{n} \leqq s} \lambda_{n}^{-1}-\frac{1}{2 \rho} \log s\right\}=-\infty .
$$

We remark that in all the above results the conclusion that $f(z)$ is unbounded on $z>0$ may be replaced by the assertion that there is no polynomial which majorises $f(z)$ on $z>0$.

2. Proofs of sufficiency. In this section we show that the gap conditions given are sufficient that $f(z)$ be unbounded on $z>0$.

For an entire function $F(z)$ which satisfies $F(0)=0$ we introduce the notation

$$
\|F\|_{x}=\left\{\int_{0}^{x}|F(x)|^{2} \frac{d x}{x^{2}}\right\}
$$

We require three lemmas, the first of which is a variant of Lemma 1 of [1].

LEMmA 1. Let $0<\mu_{1}<\mu_{2}<\ldots<\mu_{n}$. Then, for each $v=1,2, \ldots n$, there exists a realvalued function

such that

$$
B_{v}(x)=\sum_{j=1}^{n} \beta_{j, v} x^{\mu j}
$$
(i) $\int_{0}^{1} x^{\alpha} B(x) \frac{d x}{x}=\frac{1}{\alpha+\mu_{v}} \prod_{\substack{j=1 \\ j \neq v}}^{n} \frac{\alpha-\mu_{j}}{\alpha+\mu_{j}}$,
(ii) $\int_{0}^{1} B_{v}^{2}(x) \frac{d x}{x}=\left(2 \mu_{v}\right)^{-1}$. 
LEMMA 2. Let $\Lambda=\left\{\lambda_{j}\right\}$ be a set of positive integers and define

$$
\sigma(x)=\exp \left\{2 \sum_{\lambda j \leqq x} \mu_{j}^{-1}\right\}
$$

where $\mu_{j}=\lambda_{j}-\frac{1}{2}(j=1, \ldots n)$. Then there exist constants $C(\lambda)(\lambda \in \Lambda)$ which are independent of $x$ and such that the coefficients of each polynomial $P(t)$ of the form

satisfy the inequality

$$
P(t)=\sum_{\lambda_{j} \leqq x} A_{j} t^{\lambda_{j}}
$$

$$
\left|A_{v}\right| \leqq C\left(\lambda_{v}\right)\|P\|_{\sigma(x)} \quad\left(\lambda_{v} \leqq x\right) .
$$

Proof of Lemma 2. We write $\sigma$ for $\sigma(x)$ and let $P(t)=t^{\frac{1}{2}} Q(t)$. Then, by Lemma 1(i),

$$
\begin{aligned}
\int_{0}^{\sigma} Q(t) B_{v}\left(\frac{t}{\sigma}\right) \frac{d t}{t} & =\sum_{j=1}^{n} A_{j} \int_{0}^{\sigma} t^{\mu_{j}} B_{v}\left(\frac{t}{\sigma}\right) \frac{d t}{t} \\
& =\sum_{j=1}^{n} A_{j} \sigma^{\mu_{j}} \int_{0}^{1} t^{\mu_{j}} B_{v}(t) \frac{d t}{t} \\
& =A_{v} \frac{1}{2 \mu_{v}} \prod_{\substack{\lambda_{i} \leqq x \\
i \neq v}} \frac{\mu_{v}-\mu_{i}}{\mu_{v}+\mu_{i}} \exp \frac{2 \mu_{v}}{\mu_{i}} .
\end{aligned}
$$

On employing the inequality $e^{y}(1-y)<(1+y)(0<y<1)$, we deduce that

$$
\left|A_{v}\right| \leqq 2 \mu_{v}\left\{\prod_{\substack{i=1 \\ i \neq v}}^{\infty}\left|\frac{\mu_{v}+\mu_{i}}{\mu_{v}-\mu_{i}}\right| \exp \left(-\frac{2 \mu_{v}}{\mu_{i}}\right)\right\} \int_{0}^{\sigma}\left|Q(t) B_{v}\left(\frac{t}{\sigma}\right)\right| \frac{d t}{t}
$$

The convergence of the infinite product follows from that of the infinite series $\sum_{j=1}^{\infty} \mu_{j}^{-2}$. The conclusion of Lemma 2 may now be deduced by means of Schwarz's inequality and Lemma 1(ii).

Lemma 3. Suppose that $f(z) \in E(\Lambda)$ and has a power series expansion of the form (1). Suppose also that (9) and (10) hold. Then, for each fixed $R>0$,

$$
\liminf _{x \rightarrow \infty} \max _{0 \leqq t \leqq R \sigma(x)}\left|\sum_{k>x} a_{k} t^{k}\right|=0
$$

where $\sigma(x)$ is defined as in Lemma 2.

Proof of Lemma 3. For each $r>0$,

$$
\begin{aligned}
\left|a_{k}\right| & \leqq M(r) r^{-k} \\
& =\exp \{\phi(s)-s k\} \\
& \leqq \exp \{A \psi(s)-s k\},
\end{aligned}
$$


where $A$ is an appropriate constant and $r=e^{s}$. On minimising the right hand side with respect to $s$, we obtain

$$
\left|a_{k}\right| \leqq \exp \left\{-A \tau_{\psi}\left(\frac{k}{A}\right)\right\},
$$

where $\tau_{\psi}(s)$ is defined by (7). Since $\tau_{\psi}(s)$ is a convex function of $s, s^{-1} \tau_{\psi}(s)$ increases. Hence, for $0 \leqq t \leqq R \sigma(x)$,

$$
\begin{aligned}
\left|\sum_{k>x} a_{k} t^{k}\right| & \leqq \sum_{k>x}\left\{R \sigma(x) \exp \left[-\frac{A}{k} \tau_{\psi}\left(\frac{k}{A}\right)\right]\right\}^{k} \\
& \leqq \sum_{k>x}\left\{R \sigma(x) \exp \left[-\frac{A}{x} \tau_{\psi}\left(\frac{x}{A}\right)\right]\right\}^{k} .
\end{aligned}
$$

It follows from (10) that, for each fixed $R>0$, the expression inside the braces is smaller than any pre-assigned positive constant for a set of values of $x$ which is unbounded above. This completes the proof of Lemma 3.

Proof of sufficiency in Theorem 1. We fix $\lambda \in \Lambda$ and apply Lemma 2 to the function

where $\lambda \leqq x$. We obtain that

$$
P(t)=\sum_{k \leqq x}\left(a_{k} R^{k}\right) t^{k}
$$

$$
\left|a_{\lambda} R^{\lambda}\right| \leqq C(\lambda)\|P\|_{\sigma(x)}
$$

Thus

$$
\begin{aligned}
\left|a_{\lambda} R^{\lambda-\frac{t}{2}}\right| & \leqq C(\lambda)\left\|\sum_{k \leqq x} a_{k} t^{k}\right\|_{R \sigma(x)} \\
& \leqq C(\lambda)\left\{\|f\|_{R \sigma(x)}+\left\|\sum_{k>x} a_{k} t^{k}\right\|_{R \sigma(x)}\right\} .
\end{aligned}
$$

Allowing $x \rightarrow+\infty$ through a suitable sequence of values and employing Lemma 3, we obtain that

$$
\begin{aligned}
\left|a_{\lambda} R^{\lambda-\frac{1}{2}}\right| & \leqq C(\lambda) \limsup _{x \rightarrow \infty}\|f\|_{R \sigma(x)} \\
& \leqq C(\lambda)\left\{\int_{0}^{\infty}|f(x)|^{2} \frac{d x}{x^{2}}\right\}^{\frac{1}{2}} .
\end{aligned}
$$

But, if $f(x)$ is bounded on $x>0$, the right hand side is finite. A contradiction then follows on letting $R \rightarrow \infty$, unless $a_{\lambda}=0$. But this cannot be true for every value of $\lambda \in \Lambda$ because $f(z)$ is transcendental. Thus $f(x)$ is unbounded on $x>0$ and we have proved that the gap condition of Theorem 1 is sufficient.

Proof of sufficiency in Corollary 1. The inequality

$$
\tau_{\psi}(s) \geqq s \psi^{-1}(s)-s
$$


is obtained from (7) on taking $t=\psi^{-1}(s)$. The gap condition (10) is therefore implied by

$$
\liminf _{s \rightarrow \infty}\left\{2 \sum_{\lambda_{n} \leqq s} \lambda_{n}^{-1}-\psi^{-1}(s)\right\}=-\infty,
$$

which, in turn, is implied by

Suppose now that

$$
\liminf _{s \rightarrow \infty} \frac{1}{s_{\lambda_{n} \leqq \psi(s)}} \sum_{n} \lambda_{n}^{-1}<\frac{1}{2}
$$

$$
\liminf _{s \rightarrow \infty} \frac{1}{s_{\lambda_{n}} \leq \psi(s)} \lambda_{n} \lambda^{-1}<\frac{1}{2 \rho}
$$

as in Corollary 1. Then, for a suitable $\tau>\rho$,

$$
\liminf _{s \rightarrow \infty} \frac{1}{s_{\lambda_{n}} \leq \psi(t s)} \lambda_{n} \lambda^{-1}<\frac{1}{2}
$$

which is inequality (15) with $\psi(s)$ replaced by $\psi(\tau s)$. Moreover, since $f(z)$ has $\psi$-order $\rho$,

$$
\phi(s, f)=O(\psi(\tau s)) \quad(s \rightarrow+\infty),
$$

which is equation (9) with $\psi(s)$ replaced by $\psi(\tau s)$. It then follows from Theorem 1 with $\psi(s)$ replaced by $\psi(\tau s)$ that $f(z)$ is unbounded on $z>0$.

Proof of sufficiency in Corollary 2. Suppose that

$$
\limsup _{s \rightarrow \infty} \frac{1}{s_{\lambda_{n} \leqq \psi(s)}} \lambda_{n} \lambda^{-1}<\frac{1}{2 \rho}
$$

as in Corollary 2. Then, for an appropriate $\tau>\rho$,

$$
\limsup _{s \rightarrow \infty} \frac{1}{s_{\lambda_{n}} \leq \psi(r s)} \lambda_{n}^{-1}<\frac{1}{2} .
$$

But, since $f(z)$ has lower $\psi$-order $\rho$, the inequality $\phi(s)<\psi(\tau s)$ holds for a set of values of $s$ which is unbounded above. It follows that

$$
\liminf _{s \rightarrow \infty} \frac{1}{s} \sum_{\lambda_{n} \leqq \phi(s)} \lambda_{n}^{-1}<\frac{1}{2}
$$

Since $f(z)$ has $\phi$-order 1, it follows from Corollary 1 that $f(z)$ is unbounded on $z>0$.

Proof of sufficiency in Corollary 3. We take $\psi(s)=e^{\rho s}$ in Theorem 1. The equality (10) holds in this case, as can be seen by writing $\psi(s)=e^{\rho s}$ in (14). Moreover, since $f(z)$ has finite order $\rho$ and finite type,

$$
\phi(s, f)=O\left(e^{\rho s}\right)
$$

and so condition (9) holds. Theorem 1 then yields that $f(z)$ is unbounded on $z>0$. 
3. Proofs of necessity. In this section we use a construction discussed at length in [2] and [4]. If $\Lambda$ is a given set of positive integers we define the function $F(z)$ by

$$
F(z)=\sum_{n=1}^{\infty}\left\{\frac{G\left(-\lambda_{n}\right)}{\left(1+\lambda_{n}\right)^{2} G\left(\lambda_{n}\right)}\right\} z^{\lambda_{n}},
$$

where $G(z)$ is defined by

$$
G(z)=\prod_{n=1}^{\infty}\left(1-\frac{z}{\lambda_{n}}\right) \exp \frac{z}{\lambda_{n}} .
$$

This is the notation used by Edrei in [2]. The function $F(z) \in E(\Lambda)$ and

$$
F(x) \rightarrow 0 \quad(x \rightarrow+\infty) .
$$

The proof is precisely as in Section 7 of [2].

Fuchs [3] has given an estimate for the coefficients in (16). He shows that they are majorised by

$$
\exp \left\{A \lambda_{n}-2 \lambda_{n} \sum_{k=1}^{n} \lambda_{k}^{-1}\right\},
$$

where $A$ is a constant depending only on $\Lambda$. We let $F_{0}(z)=F(\gamma z)$ where $\gamma=\exp \{-(a+A+1)\}$, $a$ being a real constant to be chosen later. Then $F_{0}(z) \in E(\Lambda)$ and is bounded on $z>0$. Moreover

where

$$
\phi\left(s, F_{0}\right)=O\left(\max _{x}\{(s-a) x-x \lambda(x)\}\right)
$$

$$
\lambda(x)=2 \sum_{\lambda_{k} \leqq x} \lambda_{k}^{-1}
$$

Proof of necessity in Theorem 1. Suppose that (10) does not hold. Then, for an appropriate constant $b$,

$$
\lambda(x)-\frac{\tau_{\psi}(x)}{x}>-b
$$

for all $x>0$. Thus

$$
\max _{x}\{(s-a) x-x \lambda(x)\} \leqq \max _{x}\left\{(s-a+b) x-\tau_{\psi}(x)\right\}=\psi(s-a+b)
$$

by (8). Thus, if $a=b$, the function $F_{0}(z) \in E(\Lambda)$, is bounded on $z>0$ and satisfies (9). Thus (10) is a necessary condition.

Proof of necessity in Corollaries 1 and 2. We take $a=0$ in (17). Now

$$
\max _{x}\{s x-x \lambda(x)\}=\max _{x}\{x(s-\lambda(x))\} \leqq \lambda^{-1}(s) s,
$$


because the maximum is evidently attained for a value of $x$ which satisfies $\lambda(x)<s$. Since $\lambda(s)$ is a step function, the notation $\lambda^{-1}(s)$ needs some explanation. We define

$$
\lambda^{-1}(s)=\sup \{x \mid \lambda(x)<s\}
$$

Suppose that

$$
\liminf _{s \rightarrow \infty} \frac{1}{s_{\lambda_{n}} \leqq \psi(s)} \sum_{n} \lambda^{-1} \geqq \frac{1}{2 \rho},
$$

as in Corollary 1. Then, given any $\tau>\rho$,

$$
\lambda(\psi(\tau s)) \geqq s
$$

for all large values of $s$. Hence,

$$
\psi(\tau s) \geqq \lambda^{-1}(s)
$$

for all large $s$. Also, by (6), given any $\varepsilon>0$,

$$
\psi(\varepsilon s) \geqq s
$$

for all large $s$. Since $\log \psi(s)$ is a convex function of $s$

$$
s \lambda^{-1}(s) \leqq \psi(\varepsilon s) \psi(\tau s) \leqq \psi((\varepsilon+\tau) s)
$$

for all large $s$. It now follows, from (18), that $F_{0}(z)$ defined above has $\psi$-order $\rho$. Since, moreover, $F_{0}(x) \rightarrow 0 \quad(x \rightarrow \infty)$ this proves that the gap condition in Corollary 1 is necessary.

A similar argument suffices to prove the necessity of the gap condition in Corollary 2.

Proof of necessity in Corollary 3. It is not difficult to show that, if $\log \psi(s)$ is convex, then condition (10) is equivalent to the condition

$$
\underset{s \rightarrow \infty}{\liminf }\left\{2 \sum_{\lambda_{n} \leqq s} \lambda_{n}^{-1}-\psi^{-1}(s)\right\}=-\infty .
$$

If we take $\psi(s)=e^{\rho s}$, the necessity of the gap condition in Corollary 3 follows from that of the gap condition in Theorem 1 .

The function $f(z)=z^{-\rho} \sin z^{\rho}$ (with $2 \rho$ a positive integer) is an example of an entire function for which $f(x) \rightarrow 0 \quad(x \rightarrow \infty)$ and such that

$$
2 \sum_{\lambda_{n} \leqq s} \lambda_{n}^{-1}-\frac{1}{2 \rho} \log s=O(1) \quad(s \rightarrow+\infty) .
$$

A suitable modification yields a simpler proof of the necessity of the gap condition in Corollary 3 in the case $\lambda_{n}=2 n \rho(n=1,2, \ldots)$.

In conclusion, it is a pleasure to thank the referee for his very helpful comments. 


\section{REFERENCES}

1. J. M. Anderson and K. G. Binmore, Coefficient estimates for lacunary power series and Dirichlet series I, Proc. London Math. Soc. (3) 18 (1968), 36-48.

2. A. Edrei, Gap and density theorems for entire functions, Scripta Math. 23 (1957), 1-25.

3. W. H. J. Fuchs, On the closure of $\left\{e^{-t} t^{a} v\right\}$, Proc. Cambridge Philos. Soc. 42 (1946), 91-105.

4. A. J. Macintyre, Asymptotic paths of integral functions with gap power series, Proc. London Math. Soc. (3) 2 (1952), 286-296.

5. S. Mandelbrojt, Séries adhérentes, Régularisation des suites, Applications (Paris 1955).

6. G. Pólya, Untersuchungen über Lücken und Singularitäten von Potenzreihen, Math. Z. 29 (1929), 549-640.

UNIVERSITY COLLEGE

LONDON SCHOOL OF ECONOMICS

LONDON 